\title{
Correspondence
}

\section{Antiestrogenic drugs and atracurium - a possible interaction?}

To the Editor:

We read with interest the case report of BizzarriSchmid and Desai describing a case of prolonged neuromuscular blockade with atracurium. ${ }^{\prime}$ We have had a similar experience which we think is worth reporting.

A 67-year-old female patient weighing $57 \mathrm{~kg}$ was scheduled to undergo mastectomy for carcinoma of the breast. Preoperatively, she had hypertension which was controlled by oral methyldopa $250 \mathrm{mg}$ and hydrochlorothiazide $25 \mathrm{mg}$, twice daily and triamterene $50 \mathrm{mg}$. She was also receiving tamoxifen (an antiestrogen) $10 \mathrm{mg}$ orally twice daily as palliative treatment for breast cancer. Preoperative investigations including haemoglobin, serum electrolytes, BUN, liver function tests, ECG, chest $x$-ray and lung function tests were normal. She was premedicated with $10 \mathrm{mg}$ diazepam orally $\mathbf{9 0}$ minutes preoperatively.

Blood pressure was monitored every five minutes and the ECG, nasopharyngeal temperature and end-tidal $\mathrm{CO}_{2}$ were monitored continuously. Neuromuscular activity was monitored by recording the response of the adductor pollicis to supramaximal stimulation of the ulnar nerve using a Myotest peripheral nerve stimulator and a neeuromuscular function analyzer (Myograph 2000, Biometer). Four square wave impulses of $0.2 \mathrm{~ms}$ duration and $2 \mathrm{~Hz}$ frequency repeated every ten seconds (TOF) were employed.

After induction of anaesthesia with phenoperidine $2 \mathrm{mg}$ and thiopentone $300 \mathrm{mg}$, atracurium 28.5 $\mathrm{mg}\left(0.5 \mathrm{mg} \cdot \mathrm{kg}^{-1}\right)$ was administered and the trachea was intubated following complete suppression of the twitch response. This occurred after 145 seconds. Anaesthesia was maintained with 70 per cent nitrous oxide in oxygen and $0.5 \mathrm{mg}$ phenoperidine doses, as required. The nasopharyngeal temperature remained above $36^{\circ} \mathrm{C}$ and the lungs were ventilated to maintain normocapnoia. Apart from a brief period of hypotension following induction, the cardiovascular system remained stable throughout surgery.

It took 86 minutes for the first twitch of the TOF to reappear after the initial dose of atracurium. The expected time for retum is $36.9 \pm 8.6$ minutes. ${ }^{2}$ No further doses of atracurium were given. Surgery lasted for 140 minutes and the TOF ratio at this stage was 0.33 . The residual neuromuscular block was reversed with neostigmine $2.85 \mathrm{mg}$ and atropine $1.4 \mathrm{mg}$. Reversal was rapid and after 85 seconds the TOF ratio had reached 0.7 . The patient was extubated and sent to the recovery room. Further recovery was uneventful.

The explanation of Bizzarri-Schmid and Desai ${ }^{1}$ that the prolonged recovery from the effects of the atracurium in their patient was due to cumulation of the drug is unlikely. The non-cumulative properties of atracurium has been substantiated in several studies $^{3-5}$ and even in patients with renal or hepatic disease, the effect of atracurium has not been found to be cumulative. ${ }^{4-6}$

However, it is of interest that our patient was receiving tamoxifen, which is an antiestrogenic drug and that the patient reported by BizzarriSchmid and Desai ${ }^{1}$ was receiving danazol. Tamoxifen acts by competing with estrogen in target tissues whereas danazol decreases ovarian estrogen production by inhibiting the output of pituitary gonadotropins. There are no reports of interaction between tamoxifen and non-depolarizing muscle relaxants, but in view of our experience and that of BizzarriSchmid and Desai, 'the interaction between atracurium and antiestrogenic drugs warrants further studies. Meanwhile we consider it prudent to recommend that atracurium should be used with care in patients receiving antiestrogenic drugs.

Mohamed Naguib MB B CH M SC FFARCSI

Henry K. Gyasi MB BCH FFARCSI

Department of Anaesthesiology

King Faisal University

King Fand Hospital

P.O. Box 2208

Alkhobar 31952

Saudi Arabia 
REFERENCES

1 Bizzarri-Schmid MD, Desai SP. Prolonged neuromuscular blockade with atracurium. Can Anaesth Soc J 1986; 33: 209-12.

2 Naguib M, Gyasi HK, Abdulatif M, Absood GH. Rapid tracheal intubation with atracurium. A comparison of priming intervals. Can Anaesth Soc J 1986; 33: 150-6.

3 Basta SJ. Ali HH, Savarese JJ et al. Clinical pharmacology of atracurium besylate (BW 33A): a new non-depolarizing muscle relaxant. Anesth Analg 1982; 61: 723-9.

4 Miller RD, Rupp SM, Fisher DM, Cronnelly $R$, Fahey MR, Sohn YJ. Clinical pharmacology of vecuronium and atracurium. Anesthesiology 1984; 61: 444-53.

5 Hughes $R$. Atracurium-the first years. Clinics in Anesthesiology 1985; 3: 331-45.

6 Gyasi $H K$, Naguib $M$. Atracurium and severe hepatic diseuse. Can Anaesth Soc J 1985; 32: 161-4.

\section{Prolonged neuromuscular blockade with atracurium}

To the Editor:

I read with interest the case report by BizzarriSchmid and Desai entitled "Prolonged neuromuscular blockade with atracurium."1 The case suggests that peripheral nerve stimulation may be unreliable as a monitor of neuromuscular blockade, and it highlights the need for adequate assessment of recovery. However, I think that the patient received higher than usual doses of atracurium and the way the neuromuscular function was monitored was not entirely appropriate.

As far as I can ascertain from the text, the total amount of atracurium given was $135 \mathrm{mg}(2.3$ $\mathrm{mg} \cdot \mathrm{kg}^{-1}$ ), not $105 \mathrm{mg}$ as mentioned in the abstract, over 2.5 hours. This is considerably more $(0.9$ $\left.\mathrm{mg} \cdot \mathrm{kg}^{-1} \cdot \mathrm{h}^{-1}\right)$ than typical maintenance doses $(0.3-$ $\left.0.5 \mathrm{mg} \cdot \mathrm{kg}^{-1}\right){ }^{2}$ In addition, the patient received four drugs expected to potentiate neuromuscular blockade: gentamycin, d-tubocurarine, succinylcholine, ${ }^{3}$ and enflurane. However, some patients are unusually resistant to neuromuscular blockers, and relatively high doses of atracurium may be justified if proper monitoring is used.

The authors mentioned that they stimulated both ulnar and facial nerves, but they do not say which response they evaluated as a guide to administration of more relaxant. The muscles supplied by the facial nerve are more resistant to relaxants than the adductor pollicis. ${ }^{4}$ Thus, if the response to stimulation of the facial nerve is used as a guide, an overdose of a neuromuscular blocking drug may be given. In addition, it is not clear how the response was assessed. It has been shown that even trained observers can grossly overestimate the degree of response to train-of-four stimulation. ${ }^{5}$ Therefore, in the absence of recording equipment, it appears impossible to rule out the presence of fade.

More important, however, is the authors' use of tetanic stimulation. During the case, all necessary information could have been provided by the response to train-of-four stimulation, and the confounding effect of post-tetanic potentiation could have been avoided. ${ }^{6}$ More specifically, the use of tetanic stimulation is inappropriate before the administration of neostigmine, because this leads to an overestimate of neuromuscular recovery when further stimulation is applied. This could explain the apparent discrepancy between the response to peripheral nerve stimulation and clinical assessment. Similarly, there is no reason to apply tetanic stimulation before giving an additional maintenance dose of atracurium; the response to train-offour can provide adequate information.

I conclude that the prolonged weakness reported in this case was observed after large doses of atracurium, and its neuromuscular origin has not been firmly established.

François Donati PH D MD FRCPC

Department of Anaesthesia

Royal Victoria Hospital

and McGill University

Montreal, Quebec H3A 1AI

\section{REFERENCES}

1 Bizzarri-Schmid MD, Desai SP. Prolonged neuromuscular blockade with atracurium. Can Anaesth Soc J 1986; 33: 209-12.

2 Gramstad L, Lilleaasen $P$. Neuromuscular blocking effects of atracurium, vecuronium and pancuronium during bolus and infusion administration. $\mathrm{Br} \mathrm{J}$ Anaesth 1985; 57: 1052-9.

3 Stirt JA, Katz RL, Murray AL, Scheh DL, Lee C. Modification of atracurium blockade by halothane 